\title{
Niger: Die neue Verfassung der III. Republik
}

\author{
Von Volker Lohse
}

\section{Einführung}

Der "vent de démocratie", der seit zwei Jahren in Afrika - besonders in den francophonen Staaten - weht, hat auch die Republik Niger (RN) 1 erfaßt. Nach den Jahren der Regime von Hamani Diori und General Seyni Kountché (militärischer Staatsstreich gegen Diori am 15.4.1974) folgte 1987 die vergleichsweise milde Militär-/Einparteienherrschaft des Brigadegenerals Ali Saibou. Alle diese Regime hatten gemeinsam, daß sie nicht fähig waren, die großen Probleme des saharo-sahelischen Landes - wirtschaftliche Schwäche, Naturkatastrophen (besonders die große Dürre der 1970er Jahre), Rechtsbrüche großen Stils, Nepotismus, Bereicherung an Staatseigentum, Spannungen zwischen den Ethnien, Regionen und politischen Strömungen, Analphabetismus - zu bewältigen. Auslösende Elemente, die die Krise der von Kountché verordneten Société de Développement und das Einparteiensystem (Mouvement National pour la Société de Développement [MNSD]) 2 verdeutlichten, waren schließlich die Massaker der Sicherheitskräfte an demonstrierenden Studenten in Niamey (9.2.1990) und an Tuaregs in Tchin-Tabaraden (6./7.5.1990). Von außen animierte der französische Präsident François Mitterrand mit seiner Rede auf dem französisch-afrikanischen Gipfeltreffen von La Baule im Juni 1990 die afrikanischen Partner zu verstärkten Demokratisierungsanstrengungen, für die er verstärkte Hilfe zur Entwicklung in Aussicht stellte.

Das Staatsgebiet des heutigen Niger gehörte früher als Territoire Militaire bzw. als Colonie du Niger zu Französisch-Westafrika (Afrique Occidentale Française [AOF]); am 18. Dezember 1958 wurde das Territorium zur Republik Niger. Noch vor der formellen Unabhängigkeit des Landes am 3. August 1960 erklärte sich die Territorialversammlung 3 in Niamey zur Verfassunggebenden Versammlung 4 und verabschiedete mit 44 gegen vier Stimmen die (erste) Verfassung vom 25. Februar 1959 (Joumal Officiel [JO] spécial, S. 1,

1 Amtliche Bezeichnung: République du Niger. Vgl. zum Land: Statistisches Bundesamt, Länderbericht Niger 1992, und Robert, Dieter, Niger, in: Nohlen / Nuscheler (Hrsg.), Handbuch der Dritten Welt, Bonn, 3. Aufl. 1993, Band 4.

2 Salifou, André, Histoire du Niger, Paris 1989, passim. Der Gründungskongreß der sogar in den Verfassungstext aufgenommenen Einheitspartei (offiziell als "Bewegung" bezeichnet) fand vom 15.-18.5.1989 statt.

3 Assemblée Territoriale.

4 Assemblée Constituante. 
verkündet am 12. März 1959). Deren Text etablierte in 65 Artikeln ein auf den Regierungschef 5 und die Gesetzgebende Versammlung6 zugeschnittenes System mit mehreren politischen Parteien, aber ohne das Amt eines Staatspräsidenten und ausgeformten Grundrechtsteil.

Bereits wenige Wochen nach der Unabhängigkeit trat die (zweite) Verfassung vom 8. November 1960 (JO spécial, S. 1) 7 in Kraft. Sie sah in 76 Artikeln einen direkt gewählten, starken Staatspräsidenten ${ }^{8}$, konkret: Diori, eine schwache Regierung unter seiner Leitung und eine relativ schwache Nationalversammlung9 vor. Bezüglich der Grundrechte wurde nach französischem Vorbild wie in vielen Staaten der Französischen Gemeinschaft10 - auf die Erklärung der Menschen- und Bürgerrechte von 1789 und auf die Allgemeine Erklärung der Menschenrechte von 1948 Bezug genommen. De facto regierte Diori mti seiner Einheitspartei 11. Der Militärputsch vom 15. April 1974 (Kountché) suspendierte die Verfassung; der Ausnahmezustand12 begann. Die Organisation der Staatsgewalt erfolgte durch die Proklamation vom 15. April 1974 im Namen des Obersten Militärrats13 und durch die Verordnung vom 22. April $1974^{14}$ mit der Bildung einer provisorischen Regierung. Kountché bestätigte die faktische Beseitigung des Mehrparteiensystems durch Diori. Als Parlamentsersatz emannte Präsident Kountché am 3. August 1983150 Mitglieder des Nationalrats für Entwicklung15; sie kamen aus Berufs-, Frauen- und Jugendverbänden, islamischen Vereinigungen, dem Kreis der traditionellen Chefs (Häuptlinge) und dem Militär. Die Alleinherrschaft Kountchés wurde als "Entwicklungsgesellschaft"16 getarnt. Erst 15 Jahre nach dem Militärputsch folgte - nach dem tastenden Schritt einer Nationalcharta17 am 14. Juni 198718 - mit der Annahme der (dritten) Verfassung vom 24. September 1989 (JO

5 Président du Conseil.

6 Assemblée Législative.

7 Zu deren Änderungen s. République du Niger: Répertoire général des textes (1969), S. 47, dto. femer: Mise à jour (1975), S. 1.

8 Président de la République.

9 Assemblée Nationale.

10 Communauté Française.

11 Pari Progressiste Nigérien / Rassemblement Démocratique Africain (PPN-RDA).

12 Régime d'exception.

13 Conseil Militaire Suprême.

14 Nr. 74-1 (JO Nr. spécial 4, S. 1, geändert durch die Verordnung (Ordonnance) Nr. 83-04 vom 24. Januar 1983 (JO S. 60).

15 Conseil National de Développernent.

16 Société de Développernent.

17 Charte Nationale.

18 Angenommen durch Referendum mit einer Mehrheit von 99,64 \% (von 96,25\% der Wahlberechtigten), in Kraft getreten am 14.9.1987 (Ordonnance Nr. 87-29 portant publication de la Charte Nationale [JO S. 985], geändert durch Gesetz Nr. 91-01 v. 24. April 1991 [JO S. 494]). 
spécial Nr. 3, S. 39 ff.) ${ }^{19}$ durch Volksentscheid20 der Versuch einer konstitutionellen Neuordnung; die II. Republik entstand. Die Verfassung von 1989 installierte ein Präsidialsystem (mit Aufrechterhaltung einer politischen Rolle der Armee)21. Der Obere Rat der Nationalen Orientierung22 lenkte unter Leitung des Präsidenten den Staat und die Einheitspartei, die "Nationale Bewegung für die Entwicklungsgesellschaft"23. Die Rechtsstaatlichkeit war nur schwach ausgeprägt. Grundrechte wurden in einem Artikel aufgezählt und unter einen allgemeinen Gesetzesvorbehalt gestellt. Im Sinne verstärkter Demokratisierung wurden die 118 Artikel der Verfassung von 1989 am 24. April 1991 (JO spécial Nr. 1, S. 1) wesentlich geändert. Diese Änderung hatte der 38-köpfige Ad hoc-Ausschuß erarbeitet, der durch Erlaß vom 18. September 1990 (JO S. 697) bestellt worden war, um die Nationalcharta und die Verfassung den geänderten sozio-politischen Verhältnissen im Niger anzupassen. Der Ausschluß sollte die von Präsident Saỉbou eingeleitete Politik der "Entkrampfung" ("Décrispation") verfassungsrechtlich umsetzen.

\section{Entstehung der Verfassung von 1992}

Mit Erlaß des Präsidenten der Republik vom 4. Mai 1991 (JO S. 298)24 wurde nach dem Vorbild der Republik Benin im Niger die Vorbereitungskommission für die Nationalkonferenz (NK) 25 bestellt, die u.a. Repräsentanten der "institutions établies", d.h. von Gewerkschaften, Arbeitgeberverbänden und provisorisch zugelassenen Parteien26, umfaßte.27

Die NK trat am 29. Juli 1991 in Niamey zusammen. Präsident Saỉbou wies in seiner Eröffnungsrede darauf hin, daß die Konferenz vom ganzen Volk sehnsüchtig erwartet worden sei und daß alle Hoffnungen auf ihr ruhten. Er sei sich bewußt, daß sich die NK für souverän

19 Geändert durch Gesetz Nr. 91-02 vom 24. April 1991 (JO Nr. spécial 1, S. 1), dazu näher Raynal, Jean-Jacques, Constitutions et Régimes politiques du Niger, Niamey 1991, S. 24 ff.

20 Mehrheit von $98,28 \%$ (von $94,86 \%$ der Wahlberechtigten).

21 Ausführlich dazu: Raynal, Jean-Jacques, La IIè République du Niger: Une démocratie bien ordonnée, in: Penant 1990, S. 379 (382, 386 ff.).

22 Conseil Supérieur d'Orientation Nationale.

23 Mouvement National pour la Société de Développement (MNSD).

24 Décret Nr. 91-075 PRN.

25 Conférence Nationale (CN, JO S. 509).

26 Das Mehrparteiensystem führte das Gesetz über die politischen Parteien (Loi Nr. 91-05) vom 20.5.1991 (JO S. 358) wieder ein. Die NK erließ am 3.11.1991 durch den (gesetzesvertretenden) Akt Nr. XXIV/CN (Acte Fondamental, portant Charte des Partis politiques, JO Nr. spécial 6, S. 2) das neue Recht der politischen Parteien; dazu näher: Laouel, Commentaire et analyse de la Charte des Partis politiques, in: Sahel Dimanche v. 24.7.1992, S. 4 - s.a. FN 56.

27 Die Kommission sollte am 28.6.1991 durch einen Technischen Vorbereitungsausschuß ersetzt werden (Erlaß [Décret Nr. 91-114/PRN] über die Schaffung eines Comité technique chargé de l'organisation matérielle de la Conférence Nationale). Seine Schaffung wurde dann aber auf Drängen der Öffentlichkeit annulliert (Errlaß vom 7.7.1991 (Décret Nr. 91-119/PRN, JO S. 456). 
erklären und in einer Ubergangsphase provisorische Organe einsetzen werde. Er wende sich nicht dagegen, weil man den Lauf der Geschichte nicht aufhalten könne.28 Die NK erließ kraft ihrer Souveränität am 29. Oktober 1991 den Verfassungsvertretenden Akt Nr. XXI/ CN (JO spécial Nr. 5, S. 6) betr. die Organisation der Staatsgewalt während der Übergangsphase. Zuvor hatte die NK die Nationalcharta aufgehoben und die Verfassung suspendiert29; der Nationalrat für Entwicklung wurde aufgelöst, die Regierung abgesetzt.

Die NK wählte einen neuen Premierminister sowie den Präsidenten und die Mitglieder des Hohen Rats der Republik (HRR) 30 als Übergangsrumpfparlament. Der Präsident der Republik blieb im Amt, wurde jedoch durch den Akt Nr. XXI weitgehend entmachtet und als Inkamation der nationalen Einheit auf die Ausübung von Ehren- und Protokollfunktionen beschränkt. Die Úbergangsphase bis zur Ausübung der Staatsgewalt durch demokratisch gewählte Organverwalter in den obersten Staatsorganen setzte die NK auf 15 Monate vom 4. November 1991 bis zum 31. Januar 1993 fest.

Am 17. Januar 1992 bestellte der HRR durch Erlaß (Arrêté) 0001/PHCR die Kommission für die grundlegenden Rechtstexte (KGT) 31 und legte ihre Aufgaben fest; wichtigste Aufgabe war die Erarbeitung eines Verfassungs-Vorentwurfs für die III. Republik. Der am 7. Februar unter der Leitung ihres vom HRR gewählten Präsidenten Maïdagi Maïnassara begonnenen Arbeit der KGT lag det - unzulängliche - Entwurf des Politischen Ausschusses32 der NK zugrunde. Die KGT und vier Sachverständige berieten in zwei Unterkommissionen in Klausur in Namaro ("Verfassungskonvent von Namaro")33 den Text des 1 . Verfassungs-Vorentwurfs (VVE) und des Entwurfs des Wahlgesetzbuchs (WGBE)34 (17.-29. Februar 1992). Die Texte wurden dem Übergangsparlament am 23. März 1992 übergeben 35 und vom HRR als Arbeitsfassung akzeptiert. Der HRR ließ den Text des VVE in zwei (von zehn) Landessprachen36 übersetzen und in großer Auflage drucken. Die Medien veröffentlichten ihn auf Französisch, Rundfunk und Femsehen zusätzlich auf Haussa und Djerma.

28 Conférence Nationale Souveraine, Vol. I (1992), S. 15.

29 Die rechtliche Basis dafür bildeten der Akt (entsprechend einem Gesetz) Nr. I über die Rechtsstellung der Nationalkonferenz vom 30.7.1991 (JO Nr. spécial 2, S. 1) und der Akt Nr. III über die Souveränität der Nationalkonferenz vom 9.8.1991 (JO Nr. spécial 3, S. 1): Am 8.8.1991 erklänte sich die NK für souverän.

30 Haut Conseil de la République (HCR).

31 Commission chargée de l'élaboration des textes fondamentaux (CTF).

32 Commission politique.

33 "Les constituants de Namaro".

34 Code électoral als Zusammenf assung von nationalem und Kommunalwahlgesetz.

35 Zusätzlich wurde der Kommissionsbericht (Rapport général sur l'Avant-projet de Constiturion et Projet de Code électoral) vom März 1992 überreicht.

36 Haussa und Djerma: Diese beiden Sprachen werden von der Mehrheit der Nigrer gesprochen. 
Vom 18. August bis zum 7. September 1992 wurde er in einer Popularisierungskampagne des HRR der Bevölkerung vorgestellt, erläutert und mit ihr in allen Départements 37 und in der (kreisfreien) Communauté Urbaine de Niamey erörtert.

Die Popularisierungskommissionen bestanden aus HRR- und KGT-Mitgliedern und führten in den Départements- und Arrondissementshauptorten Informationsveranstaltungen für Multiplikatoren (z.B. Beamte, Lehrer), Volksversammlungen und Diskussionsrunden durch. Die Multiplikatoren übernahmen die Popularisierung des VVE in Dörfern und Weilem. Im Département Agadez behinderten Elemente der "Troupe", einer Art von Soldatenrat, die Arbeit der Kommission, indem er in offener Insubordination - angeblich im Kampf gegen die Rebellion - 200 Tuareg als (vermeintliche) Rebellen festnahm. Im gesamten übrigen Land verlief die Kampagne friedlich und erfolgreich.

Durch Beschluß vom 21. September 199238 setzte die HRR die Nationale Kommission für die Synthese der Zusätze und die Erweiterung des VVE (KSE) 39 ein und legte ihren Arbeitsauftrag fest: Die aus Mitgliedem der KGT und aus je zwei Vertretern der Départements bzw. Niameys bestehende KSE beriet die Anregungen aus der Bevölkerung und änderte bzw. ergänzte den VVE in 20 Artikeln zwischen dem 22. und 26. September 1992; der zweite VVE entstand. Der HRR nahm den modifizierten Text mit einigen Änderungen am 30. September 1992 als Verfassungsentwurf (VE)40 an. Im Referendum vom 26. Dezember 1992 votierten fast $90 \%$ der teilnehmenden Abstimmungsberechtigten mit Ja. Abstimmungsberechtigt waren 3900881 Nigrer. Davon haben 2207220 (=56,58\%) abgestimmt. 1945653 (= 89,79 \%) votierten mit Ja, 221267 (= 10,21 \%) mit Nein.

Dieses positive Ergebnis des Referendums ist um so bemerkenswerter, als sowohl die Tuareg-Rebellen im Norden des Landes (Front de libération de l'Air et de l'Azawak FLAA -) als auch die islamischen Fundamentalisten sich für einen Boykott der Abstimmung bzw. ein Nein-Votum ausgesprochen hatten. Erstere drohten mit Gewalt gegen Abstimmende, letztere hatten z.B. im Bereich der großen Märkte des Landes Tausende von Audiokassetten (für Analphabeten) mit der Aufforderung zum Nein und ihrer Begründung dazu verteilen lassen. Auch die katastrophale Wirtschafts- und Finanzlage des Niger sowie technische Schwierigkeiten der Organisation des Referendums (z.B. fehlende Abstimmungszettel, unzulässige Vertretungsabstimmungen [procurations], unterbesetzte Abstimmungsbüros) erschwerten die Entscheidung des Niger zum Ja.

37

38 Décision Nr. 0014/P.HCR.

39 Commission Nationale de Synthèse des Amendements et d'Enrichissernent de l'Avant-projet de Constitution de la IIIè République.

40 Projet de Constitution. 
Technisch vorbereitet hatte die Nationale Wahlkommission (Commission nationale des élections) die Volksabstimmung; überwacht wurde sie von der am 25. August 1992 gegründeten Nationalen Kontrollkommission (Commission nationale de contrôle et de supervision du référendum constitutionnel, des opérations électorales et post-électorales pendant la période de transition - COSUPEL-, JO spécial Nr. 4, S. 2).

Der Oberste Gerichtshof stellte unter dem 19. Januar 1993 das amtliche Endergebnis fest (JO spécial Nr. 1, S. 1), der Präsident der Republik fertigte den Text am 22. Januar 1993 aus. Die Verfassung wurde am 23. Januar im Amtsblatt verkündet (JO spécial Nr. 1, S. 2).

\section{Systematik}

Die 129 Artikel der Verfassung der III. Republik von 1992 (Verfassung 1992) gliedern sich in die Präambel und 14 Abschnitte:

I Der Staat und seine Souveränität41 - Art. 1 - 10

II Die Grundrechte und Grundpflichten der Menschen42 - Art. 11 - 36

III Die Exekutive43 - Art. 37 - 65

IV Die Legislative44 - Art. 66-76

V Die Beziehungen von Exekutive und Legislative45 - Art. 77-97

VI Die Úberprüfung der VerfassungsmäBigkeit von Gesetzen46 - Art. 98

VII Die Judikative47 - Art. 99 ff., 1. Der Oberste Gerichtshof - Art. 104, 2. Der Staatsgerichtshof - Art. 105-108

VIII Der Wirtschafts-, Sozial- und Kulturrat48 - Art. 109-111

IX Der Medienrat49 - Art. 112-114

X Die Gebietskörperschaften50 - Art. 115 f.

XI Die internationalen Verträge und Abkommen51 - Art. 117-120

XII Die Zusammenarbeit und die Verbindung mit anderen Staaten52 - Art. 121

41 I De l'Etat et dela Souveraineté.

42 II Des droits et des devoirs de la personne humaine.

43 III Du Pouvoir exécutif.

44 IV Du Pouvoir législatif.

45 V Des rapports entre les Pouvoirs exécutif et législatif.

46 VI Du contrôle de la constitutionnalité des lois.

47 VII Du Pouvoir judiciaire; 1 De la Cour Suprême; 2 De la Haute Cour de justice.

48 VIII Du Conseil économique, social et culturel.

49 IX Du Conseil supérieur de la communication.

$50 \mathrm{X}$ Des collectivités territoriales.

51 XI Des traités et accords intemationaux.

$52 \mathrm{XII}$ De la coopération et de l'association avec les Etats. 
XIII Die Verfassungsänderung ${ }^{53}$ - Art. 122-124

XIV Die Übergangs- und Schlußbestimmungen54 - Art. 125-129

\section{Staatssymbole und Staatsziele}

Der I. Abschnitt der Verfassung 1992 handelt - der Tradition Frankreichs und der Französischen Gemeinschaft55 folgend - vom Staat und seiner Souveränität. Fahne/Flagge, Wappen und Nationalhymne werden beschrieben (Art. 1). Als Staatsform wird die Republik festgelegt und jeder Angriff darauf als strafbarer Hochverrat qualifiziert; durch die partielle "Ewigkeitsklausel" des Art. 124 Abs. 2 wird die Staatsform von Verfassungsänderungen ausgenommen. Der wichtige Art. 4 markiert in Abs. 1 als Staatszielbestimmungen Einheit/ Unteilbarkeit, Demokratie und Sozialstaat. Bereits in der Präambel, die auch nach allgemeiner nigrischer Auffassung geltendes Verfassungsrecht ist, bekennt sich das nigrische Volk als Souverän zu pluraler Demokratie56 und Menschenrechten, zur afrikanischen Einheit und zur friedlichen und freundschaftlichen Zusammenarbeit mit allen Völkem. Mit der Annahme der Verfassung im Volksentscheid schwor es zugleich Loyalität, Treue und Achtung der Verfassung. Dieser letzte Satz der Präambel ist nicht zuletzt wegen des rapiden Verfalls der Staatsautorität während der Ubergangsphase bedeutsam; im Jahre 1992 zeigten sich als backlash zu der übersteigerten äußeren Staatsautorität der vorangegangenen Regime deutliche Erosionserscheinungen im Verhältnis Bürger - Staat, z.B. eine breite Steuerzahlungsverweigenung, auf die die Transitionsorgane hilflos reagierten.

Das Volk übt seine Trägerschaft nur in Referenden und Wahlen aus. Bezüglich der Ausübung der Staatsgewalt durch die Staatsorgane versucht Art. 5 rechtliche Sicherungen gegen individuelle Diktatur, Regionalismus, Ethnozentrismus, Nepotismus, Korruption etc. aufzubauen.

Die Demokratiekomponente als fundamentales Struknurprinzip 57 wird ausgeformt durch die Wahlmodalitäten (allgemein, frei, gleich, unmittelbar und geheim) 58 der Art. 7 f, 67 f.,

53 XIII De la Révision.

54 XIV Dispositions transitoires et finales.

55 Communauté Française.

56 Näher: Laouel, Renouveau démocratique et Référendum, in: Sahel Dimanche v. 14.8.1992, S. 4 (mangels finanzieller Mittel für Fachzeitschriften/-publikationen wird auch die juristische Diskussion derzeit in den Wochenzeitungen - Tageszeitungen existieren im Niger nicht - geführt); ders., Analyse de la notion de démocratie, in: Sahel Dimanche v. 21.8.1992, S. 4.

57 Die Demokratie wird in Art. 4 Abs. 2 als "Herrschaft des Volkes durch das Volk und für das Volk" beschrieben.

58 Die einzelgesetzlichen Regelungen dazu finden sich im Wahlgesetzbuch (WGB) (Code électoral [CE]) vorn 22.8.1992 (JO spécial Nr. 3, S. 2); dazu näher: Laouel, Commentaire et analyse du Projet de Code électoral du Niger, in: Sahel Dimanche v. 7.8.1992, S. 4. 
das Mehrparteiensystem mit der Chancengleichheit der politischen Parteien ${ }^{59}$ und dem Verbot ethnischer, regionalistischer oder religiöser Parteien des Art. 10 und Grundrechte der Kommunikation (Meinungs- und Pressefreiheit [Art. 24, 113]60, Versammlungs- und Vereinigungsfreiheit [Art. 25] sowie Gewerkschaftsfreiheit [Ar. 27]).

Der Sozialstaatsgrundsatz als im hohen Maße auslegungsbedürftiges Prinzip gilt im Niger als verbindliche Auslegungsregel und als Auftrag an den Gesetzgeber. Seine Einzelausprägungen in der Verfassung sind teilweise nur durch teleologische Auslegungen zu ermitteln, etwa beim Bildungs(grund)recht (Art. 12), Eigentums(grund)recht (Art. 22), Grundrecht auf Arbeit (Art. 26), Umwelt(grund)recht (Art. 28) sowie bei den Grundpflichten (Art. 29 ff.). Daß es beim Niger als einem der ämsten Länder der 3 . Welt61 keine verfassungsmäßigen Festlegungen von Daseinsvorsorge, Subventionen, Globalsteuerung der Wirtschaft etc. gibt, sollte nicht verwundern. Dagegen sind (einfache) Maßnahmegesetze und Programmgesetze 62 nach Art. $81 \mathrm{f}$. in verschiedenen sozialstaatlichen Bereichen vorgesehen.

Als weiteres Grundprinzip legt die Verfassung 1992 in Art. 4 Abs. 2 die Trennung von Staat und Religion63 fest; auch diese wurde in die partielle Ewigkeitsgarantie des Art. 125 Abs. 2 einbezogen. Die erst von der KSE eingeführte Formulienung von der "séparation de l'Etat et de la religion" ersetzt den von der KGT aus allen früheren nigrischen Verfassungen (und letztlich von Frankreich64) übernommenen Begriff von der "République laïque". Die islamischen Vereinigungen65, allen voran das "Collectif des Associations Islamiques", liefen Sturm gegen den Begriff der Laizität unter der polemischen Formel "Etes-vous musulman ou laïc?", d.h. mit der Unterstellung Laizität = Atheismus. Das hatte Reaktionen der kleinen, aber einflußreichen christlichen Kirchen im Niger zur Folge.66 Der Streit ist vermutlich auf die gewachsene Kampfkraft der fundamentalistischen Strömung des Islam im Niger - nicht zuletzt infolge der Ereignisse in den Nachbarstaaten Algerien, Libyen und (Nord-)Nigeria - zurückzuführen und hatte angesichts des Verbots religiöser Parteien67 wohl auch Ventilfunktion. In den Départements (außer Agadez) hatten viele Diskussionsteilnehmer während der Popularisierungskampagne dementsprechend die Streichung des Staatsziels "laïque", teilweise sogar seine Ersetzung durch "islamique" gefordert. Dem kam

5918 politische Parteien wurden bis zum November 1992 nach den Vorschriften des Parteiengesetzes (FN 26) anerkannt.

60 s.u. FN 108.

61 Least Developed Countries (LLDC) i.S.d. von den VN 1971 eingeführten Kriterienkatalogs.

62 Lois d'orientation.

63 Spezialregelungen in Art. $9 \mathrm{f}$, $24 \mathrm{f}$.

64 Art. 2 Abs. 1 der Verfassung vom 4.10.1958.

65 Etwa $97 \%$ der über 8 Millionen Einwohner des Niger sind Moslems.

66 Vgl. Allakaye, La laïcité sur le tapis de la Constitution, in: Le Républicain v. 10.9.1992, S. 5, und die Antwort von Alfari, ebda. 17.9.1992, S. 6; Gali, La laïcité, in: Le Républicain v. 1.10.1992, S. 2; Laouel, Indivisibilité et laïcité de l'Etat nigérien, in: Sahel Dimanche v. 4.9.1992.

67 durch den Akt XXIV/CN v. 3.11.1991 (JO Nr. spécial 6, S. 2). 
die KSE einerseits nach, verdeutlichte aber andererseits die Trennung von Staat und Religion. 68

Die Rechtsstaatlichkeit (Art. 9) wurde in der Verfassung 1992 deutlich ausgebaut. Normiert wurde sowohl das Rechtsstaatsprinzip als solches (La République du Niger est un Etat de droit, Art. 9 Abs. 1)69 als auch eine Reihe von Einzelelementen des Typus Rechtsstaat. Die Einzelregelungen sind teils (mehr) formell, teils (mehr) materiell akzentuiert worden. Die Gleichheit vor dem Gesetz (Art. 9 Abs. 2) und Justizgrundrechte (Art. 12 ff.), ein gestaffeltes System von Fachgerichten (Art. 99 ff.) mit unabhängigen und nur dem Gesetz unterworfenen Richtem (Art. 101) finden sich ebenso wie Gewaltentrennung (Art. 37 ff., 66 ff., 99 ff.) und (andere) Grundrechte (Art. 11 ff.). Das ausdrücklich formulierte Rückwirkungsverbot bezieht sich nur auf Strafgesetze, jedoch dürfte retroaktive Rückwirkung von anderen Gesetzen im Niger nur aus ganz speziellen, zwingenden Gründen des Gemeinwohls oder bei fehlendem schutzwürdigen Vertrauen eines Bürgers (oder Ausländers, Art. 9 Abs. 5) zulässig sein. 70

\section{Grundrechte und Grundpfichten}

Einen besonders bemerkenswerten Schritt zum bürgerfreundlichen, demokratischen, rechtsstaatlichen und sozialen Staat bedeutet der Ausbau der Grundrechte und Grundpflichten (primär) im II. Abschnitt der Verfassung 1992. Die jetzt geltende Fassung stellt gegenüber den vorangehenden Verfassungen einen qualitativen Sprung nach vome dar. Der einzelne Mensch71 wird - und das ist bei einem Staat mit fast ausschließlich islamisch geprägter Bevölkerung keine Selbstverständlichkeit - in den Mittelpunkt der Rechtsordnung gestellt: "La personne humaine est sacrée. L'Etat a l'obligation absolue de la respecter et de la protéger. Il lui garantit un plein épanouissement." (Art. 11, vgl. auch Art. 15).

Systematisch finden sich die Grundrechte - jetzt ihrer Bedeutung gemäß vorgezogen - in den Art. 11-28, die Grundpflichten in den Art. 29-35 des II. Abschnitts. Grundrechtsgleiche

68 Dafür findet sich der im Niger jetzt geänderte Begriff der Laizität in den - von der KGT generell zum Vergleich herangezogenen - Verfassungen von Benin (Ar. 2 Abs. 1), Burundi (Ar. 1 Abs. 1), Mali (Ar. 25 Abs. 1), Namibia (Ar. 1 Abs. 1 "secular") und Togo (Ar. 1); er wird üblicherweise definiert als Staat, "qui est indépendent de toute confession religieuse" (vgl. statt vieler: Cornu, Gerard, Vocabulaire juridique (31992), Paris, S. 466).

69 Die Verf assung 1992 geht damit weiter als das Grundgesetz, das den Rechtsstaat als Inbegriff der normativ belegbaren Einzelprinzipien ("im Sinne dieses GG", Ar. 28 Abs. 1 S. 1 GG) konstitutionalisierte.

70 Wie hier: Laouel, L'Avant-projet de Constitution: Inspiration, Caractères généraux, Nature du régime, in: Sahel Dimanche v. 28.8.1992, S. 4.

71 Zur grundsätzlichen Gleichstellung von Nigrem und Ausländem vgl. Art. 9 Abs. 5. 
Rechte enthalten die Art. 3 (Nationalsprachen-Freiheit), 6 (Widerstandsrecht), 8, 67 (Wahlrechtsgleichheit), 9 (allgemeiner Gleichheitssatz und grundsätzliche Gleichstellung von Nigrem und Ausländern auf dem Staatsgebiet der Republik Niger), 10 (Parteienfreiheit), $100 \mathrm{f}$. (Recht auf den gesetzlichen Richter) und 98 (Recht auf Überprüfung der Verfassungsmäßigkeit [formeller] Gesetze).

Die eigentlichen Justizgrundrechte nahm der Verfassungsgeber - und über diese Systematik kann man gewiß streiten 72 - in den Grundrechtsabschnitt auf: Art. 14 (Verbot der Folter), 16 (Rückwirkungsverbot von Strafgesetzen, Verbot der Ausweisung), 18 (Unschuldsvermutung).

Zu den klassischen Grundrechten gehören Art. 12 (Recht auf Leben und körperliche Unversehrtheit), 15 (freie Entfaltung der Persönlichkeit), 19 (Ehe und Familie), 20 (Erziehungsrecht/-pflicht der Eltem), 21 (Unverletzlichkeit der Wohnung), 23 (Brief- und Kommunikationsgeheimnis), 24 (Meinungs- und Religionsfreiheit), 25 (Vereinigungs- und Versammlungsfreiheit) und 27 (Gewerkschafts- und Streikfreiheit). 73

Aus dem gewohnten Rahmen fällt zunächst das Menschenrecht auf Bildung und Ausbildung (Art. 12), das allerdings einer (speziellen) Gesetzesschranke74 unterworfen wird und damit weitgehend leerläuft.

Art. 28 enthält teils Grundrecht, teils Programmsatz, dieses Umwelt(grund)recht erscheint überdies als zu unbestimmt: Toute personne a droit à un environnement sain (Art. 28 Abs. 1 S. 1). Speziel werden dann in Abs. 2 diesbezlglich u.a. verboten: der Import von ausländischen giftigen oder schadstoffhaltigen Abfällen. Die Lagenung, Bearbeitung oder der Abtransport entsprechender einheimischer Abfälle "werden durch (einfaches) Gesetz geregelt". Nimmt man den - französischer Rechtstradition entsprechenden - allgemeinen Gesetzesvorbehalt des Art. 3675 hinzu, bleibt von dem subjektiven Recht auf gesunde Umwelt nur ein kleiner Rest übrig. Immerhin wird durch Art. 28 (auch) der Gesetzgeber gebunden; Art. 35 folgt insoweit Art. 1 Abs. 3 GG76.

Das Widerstandsrecht (Art. 6) wird einerseits als Recht und Pflicht zu zivilem Ungehorsam gegen eine Unterdrückungsherrschaft (régime oppressif) ausgestaltet. Als solche Unrechts-

72 Vgl. Ar. 101, 103, 104 GG.

73 Eine vergleichbare verfassungsrechtliche Regelung für die Aussperrung fehlt.

74 "dans les conditions définies par la loi".

75 Tous ces droits et libertés s'exercent dans le respect des lois et règlements en vigueur.

76 Art. 35: Les droits et les devoirs ci-dessus proclamés et garantis par la présente Constitution lient les pouvoirs législatif, exécutif et judiciaire ainsi que toute personne, à titre de droit directement applicable. Bemerkenswert ist die weit gefaßte Drittwirkung, die sich in der Praxis aber erst bewähren muß. 
herrschaft definiert Art. 6 Abs. 1 S. 2 ein Regime, das absichtlich die Vorschriften der Verfassung verletzt. Andererseits hat das Volk (sachgerechter wäre es gewesen, "die Nigrer" als subjektiv Berechtigte zu nennen, weil "das Volk" als Ganzes das Recht vermutlich nicht ausüben kann) das Recht, durch zivilen Ungehorsam die demokratisch legitimierte Herrschaft gegen jeden Staatsstreich (coup d'Etat) zu verteidigen. Der zivile Ungehorsam ist nur friedlich und als letztes Mittel, d.h. wem andere Abhilfe nicht möglich ist, gestattet. Nicht nur angesichts des im Norden Nigers tobenden Tuaregaufstands fragt es sich, ob die gewählte Fassung des Widerstandsrechts nicht $\mathrm{zu}$ weit und zu ungenau ausgefallen ist.

Die Grundpflichten schließen die Verteidigungspflicht ("un devoir sacré") des Art. 30 ebenso ein wie die Verpflichtung zu öffentlichen Arbeiten und zur Erfüllung der Bürgerpflichten. Die Unverletzlichkeit der "biens publics" wird als Beachtungspflicht ("respecter scrupuleusement") und als Schutzpflicht ausgestaltet. Gegen Sabotage, Vandalismus, Korruption, Unterschlagung, Zweckentfremdung, Verschwendung und ungerechtfertigte Bereicherung daran werden einzelgesetzlich zu fassende Strafen angedroht.

Art. 34 - hier an sich unsystematisch eingefügt - begründet die Verpflichtung des Staates zur Verbreitung des Verfassungstextes und zur Unterrichtung (der Bürger) über den Verfassungsinhalt sowie über Menschenrechte und Grundfreiheiten.

\section{Exekutive}

In Art. 37-65 folgen77 die Regelungen zur vollziehenden Gewalt78. Auf die Empfehlung der Nationalkonferenz haben sich die Väter und Mütter (sieben der 49 Mitglieder der KGT waren Frauen) der Verfassung für ein semi-präsidentielles System entschieden, das nach nigrischem Verständnis charakterisiert wird durch

- einen starken, direkt gewählten Präsidenten und einen parlamentarisch verantwortlichen Premictminister sowie

- eine Nationalversammlung, die auf der Basis eines breiten Gesetzgebungskatalos legiferiert und die Tätigkeit der Regierung kontrolliert.79

77 Der Aufbau der Verfassung und der Inhalt des III. Abschnitts entsprechen weitgehend dem französischen Vorbild.

78 Näher: Laouel, Le pouvoir exécutif, in: Sahel Dimanche v. 12.6.1992, S. 4.

79 Die Halbierung der Staatsgewalt wird demnach nur auf den Präsidenten und die Nationalversammlung bezogen. Vgl. für die ganz h.M.: Rappor général sur l'Avant-projet de Constitution et Projet de Code électoral (1992), S. 3 und Laouel, FN 70, sowie ders., Les Principes généraux de l'Avant-projet de Constitution de la IIIè République, in: Sahel Dimanche v. 5.6.1992, S. 2. 
Der Präsident der Republik (PR) ${ }^{80}$, stärkerer Teil der doppelköpfigen81 Exekutive, erhält unbestreitbare Legitimation durch seine unmittelbare Wahl durch das Volk auf fünf Jahre (Art. 38).82 Als Staatschef verkörpert er die Einheit der Nation und garantiert die Achtung der Verfassung; er sichert das ordnungsgemäße Funktionieren der Staatsgewalt (art. 37). Im Verhinderungsfall vertritt ihn der Präsident der Nationalversammlung (Art. 44, 71). Der PR ernennt und entläßt den Premierminister (PM) (Art. 47); er führ den Vorsitz im Ministerrat (Art. 48). Als Staatspräsident hat er bedeutende Zuständigkeiten im außenpolitischen Bereich (Art. 37, 117 ff.). Allerdings sieht Art. 63 für einige politisch relevante Bereiche die Gegenzeichnung des Premierministers oder des zuständigen Ministers vor. Der PR fertigt die Gesetze aus und verkündet sie; zuvor kann er sogar eine emeute Beratung in der Nationalversammlung verlangen. Nach der Stellungnahme der Nationalversammlung kann er Gesetze, für die er die unmittelbare Konsultation des Volkes für nötig erachtet, einem Referendum unterwerfen. Der PR kann sich direkt oder durch zu verlesende Botschaften an die NV wenden.

Allerdings sieht Art. 63 für einige politisch relevante Bereiche die Gegenzeichnung des Premierministers oder des zuständigen Ministers vor. Die größe zu erwartende Schwierigkeit im janusköpfigen Bereich der Exekutive zeigt die Zusammenschau der Art. $53 \mathrm{f}$. und 62: Nach den beiden erstgenannten Vorschriften ist der Präsident der Republik Chef von Verwaltung und Streitleäften, nach der letztgenannten verfügt die Regierung83 über Verwaltung und Armee. Es lassen sich politische und personenbezogene Konstellationen denken - etwa in Fällen von cohabitation -, in denen es aufgrund dieser Bestimmungen zu einer Paralysierung der Exekutive kommt.

Als oberstes Staatsorgan hat die Verfassung 1992 im Niger erstmals den Premierminister mit Verfassungsrang festgeschrieben.84 Nach einer Art von Premierministerprinzip ist der PM Chef der Regierung (also deutlich mehr als ein primus inter pares im Verhältnis zu den Ministern); er bestimmt, leitet und koordiniert das Regierungshandeln, sichert die Ausführung der Gesetze (Art. 60). Die Minister werden auf seinen Vorschlag vom PR emannt und entlassen (Art. 47). Da die Regierung vom Parlament (bzw. von dessen Mehrheit) abhängt (vgl. Art. 62 Abs. 3), hatte die KGT vorgeschlagen, daß der PM vom PR nur auf seinen des PM - Regierungs-Rücktrittsvorschlag entlassen werden durfte (Art. 47 Abs. 1 a.F.). Das stellte in der von der KGT filigran ausgearbeiteten Machtbalance der Verfassung den actus contrarius zu der Designierung des PM durch die Parlamentsmehrheit dar. Der HRR hat

80 Näher: Laouel, La fonction présidentelle sous la IIIè République, in: Sahel Dimanche v. 18.9.1992, S. 4.

81 Präsident der Republik und Premierminister.

82 Er darf einmal wiedergewählt werden (Ar. 38).

83 Näher: Laouel, Le rôle du Gouvemement sous la IIIè République, in: Sahel Dimanche v. 25.9.1992, S. 4.

84 In der Vergangenheit wurde das Amt des Premierministers einmal sogar (legal) abgeschafft. 
diese Einschränkung in seiner abschließenden Beratung des VVE gestrichen und den PM neben seiner Abhängigkeit von der Nationalversammlung in eine zusätzliche Abhängigkeit vom PR gebracht. Damit wurde nicht nur im Dualismus im exekutiven Bereich, sondern auch bezüglich des semipräsidentiellen Systems eine unausgewogene Machtsteigerung des PR bewirkt. 85

Das Kollegialprinzip schreibt Art. 62 fest, nach dem die Regierung die Politik der Nation bestimmt und lenkt; der Ministerrat ruft auch den Belagerungszustand aus (Art. 86). Der Regierung stehen das Gesetzesinitiativrecht (Art. 90)86 und das Verordnungsrecht (Art. 87) zu. Die Regierungsmitglieder haben Zugang zurn und Rederecht im Parlament und seinen Ausschüssen (Art. 79).

\section{Legislative}

Der Niger hat sich im Bereich der gesetzgebenden Gewalt für ein Einkammersystem entschieden (Art. 66) 87. Die Nationalversammlung besteht aus 83 Abgeordneten, die nach dem Verhältniswahlrecht auf fünf Jahre gewählt werden (Art. 67)88. Wahlberechtigt ist grundsätzlich ${ }^{89}$ jeder Nigrer/jede Nigrerin, der/die das 18 . Lebensjahr vollendet hat und im Besitz seiner/ihrer bürgerlichen Ehrenrechte ist; ferner sind für volljährig erklärte Minderjährige aktiv wahlberechtigt (Ar. 8). Wählbar ist jeder nigrische Staatsbürger, der das 25. Lebensjahr vollendet hat und im Besitz seiner bürgerlichen Ehrenrechte ist (Art. 92 WGB). Nur politische Pateien dürfen Kandidaten für die Parlamentsmandate aufstellen.90

85 Aïchatou, Dodo, Chronique d'une instabilité annoncé, in: Sahel Dimanche v. 16.10.1992, S. 2 schreibt diesbezüglich sogar von einem "coup d'Etat" des Übergangsparlaments.

86 Neben der Regierung haben die Mitglieder der Nationalversammlung das Recht zur Gesetzesinitiative.

87 Einzelheiten s. Laouel, Le pouvoir législatif, in: Sahel Dimanche v. 19.6.1992, S. 4 und v. 26.6.1992, S. 4.

88 Einzelheiten im Wahlgesetzbuch (WGB) (Code électoral) Nr. $92-43$ v. 22.8.1992 (JO Nr. spécial 3, S. 2); das WGB regelt das Parlaments- und das Kommunalwahlrecht. Zur Analyse der nigrischen Demokratie vgl. Laouel, Commentaire et analyse du Projet de Code électoral du Niger, in: Sahel Dimanche v. 7.8.1992, S. 4.

89 Ausnahme z.B. ein wegen eines Verbrechens Verurteilter, der nicht nach Aufhebung der Straffolgen in seine früheren Rechte (wieder) eingesetz wurde (Art. 8 WGB).

90 Das Nähere regelt das Parteiengesetz, s.o. FN 26; dazu Laouel, Commentaire et analyse de la Charte des Partis politiques, in: Sahel Dimanche v. 24.7.1992, S. 4. Zum Szenario der ersten Wahlen für die III. Republik vgl. Chaibou, in: Sahel Dimanche v. 11.9.1992, S. 12. Ein Sonderproblem stellt das Verbot der Kandidatur der Spitzen der Übergangsorgane, d.h. des Präsidenten der Republik Ali Saibou, des Präsidenten des HCR André Salifou und des Premierministers Amadou Cheiffou, für die Präsidentenwahlen im Januar 1993 dar (Art. 147 WGB), das auf einen Beschluß der Nationalkonferenz beruht, vgl. Laouel, La candidature éventuelle de la Troika aux élections de décembre 1992, in: Sahel Dimanche v. 29.7.1992, S. 36. 
Ein imperatives Mandat wird ausgeschlossen (Art. 69 Abs. 2), Immunität wird garantiert. Im Fall des Todes, der Demission oder endgültiger Verhinderung der Wahmehmung des Mandats tritt ein persönlicher Ersatzkandidat an die Stelle des Deputierten (Art. 89 WGB). Dem für die Dauer der Legislaturperiode gewählten Parlamentspräsidenten steht ein gewähltes Büro (Präsidium) zur Seite. Die Grundzüge des Inhalts der zu erlassenden Geschäftsordnung fixiert Art. 76.

In einem speziellen V. Abschnitt regelt die Verfassung 1992 die Beziehungen zwischen Exekutive und Legislative einschließlich zweiter Gesetzgebungskataloge: Art. 81 enthält die Aufzählung der Gegenstände, die durch formelle Gesetze zu regeln sind; sie wurde gegenüber den früheren nigrischen Verfassungen im Interesse von Demokratie und Rechtsstaat erweitert, z.B. um ein Gesetz über das Statut der traditionellen Chefferie. Art. 82 beschreibt die Gegenstände für Rahmenvorschriften 91 , die durch Verordnungen der Exekutive auszufüllen sind (Art. 84), Art. 83 die Gegenstände der Finanzgesetzgebung. Das Gesetzgebungsverfahren wird nur rudimentär in den Art. $90 \mathrm{ff}$. festgelegt und darüber hinaus der Geschäftsordnung der Nationalversammlung überlassen (Art. 76).

Genaue Regeln stellt die Verfassung 1992 dagegen für die Kontrolle der Verfassungsmäßigkeit formeller Gesetze auf (Art. 98): Grundlegende Gesetze über Schaffung neuer oder die Umgestaltung bestehender Staatsorgane (lois organiques) sind vor ihrer Verkündung dem Obersten Gericht 92 zur Prüfung der Verfassungsmäßigkeit zu unterbreiten. Bezüglich anderer formeller Gesetze kann vor ihrer Verkündung das Oberste Gericht vom Präsidenten der Republik, vom Präsidenten der Nationalversammlung, von Premierminister oder von einem Zehntel der Abgeordneten der Nationalversammlung zur Prüfung der Verfassungsmäßigkeit angerufen werden. Eine bedeutsame Erweiterung der rechtsstaatlichen Kontrolle bringt Abs. 5 des Art. 98, der jeder Partei eines Gerichtsverfahrens ermöglicht, in einem Sonderverfahren die Nichtverfassungsmäßigkeit eines formellen Gesetzes geltend zu machen; die Verfassungswidrigkeitserklärung des angegriffenen Gesetzes beinhaltet dessen (partielle) Nichtigkeit (Art. 6).

Die Nationalversammlung kontrolliert das Regierungshandeln (Art. 72) und hat ein Zitierungsrecht gegenüber Regierungsmitgliedern; sie verfügt ferner über ein breites Spektrum parlamentarischer Anfragen (Art. 80 Abs. 2). Die Nationalversammlung muß zur Kriegserklärung ermächtigen (Art. 85).

91 Principes fondamentaux.

92 Cour Suprême. 
Der Premierminister hat das Recht, in der Nationalversammlung die Vertrauensfrage zu stellen; die Nationalversammlung kann ihm das Mißtrauen aussprechen.93

In der Kommission für die grundlegenden Rechtstexte diskutierte man auch die Frage der Einrichtung einer zweiten (Parlaments-)Kammer. Aus Sorge vor Teilseparationen94 wurde die zweite Karnmer von ihren Anhängem nicht als Föderal-, sondern als Regionalkammer gefordert. Obgleich die Schaffung einer zweiten Kammer mehrheitlich in der KGT abgelehnt wurde, griff die Stellungnahme der Regierung zum VVE (vorbereitet von Larwana/ Lohse) die Anregung zu einer Regionalkammer gerade im Hinblick auf die notwendige Nord-Süd-Entspannung im Niger noch einmal auf, und der Ministerrat empf ahl ihre weitere Diskussion im Hinblick auf eine künftige Verfassungsänderung.

\section{Judikative}

In ihrem VII. Abschnitt weist die Verfassung 92 die Justiz als dritte Gewalt, als pouvoir judiciaire, aus. Frühere nigrische Verfassungen begnügten sich für die damals weit weniger unabhängige Justiz mit der Bezeichnung autorité judiciaire.95

Die Verfassung 1992 garantiert die Unabhängigkeit der rechtsprechenden Gewalt (Art. 99, 101)96, sie führt Justizgrundrechte ein (Art. 13 ff.); ein Richterrat (Art. 103, 102)97 schützt die Richter und wirkt bei Emennungen und Befördenungen mit. Die Exekutive darf nicht (mehr) in Gerichtsverfahren intervenieren. Früher war z.B. General Kountché als Präsident der Republik für seine Eingriffe in laufende Gerichtsverfahren bekannt (auch wenn selbst unter ihm ein genereller Rückfall in die Kabinettsjustiz vergangener Jahrhunderte nicht stattfand). Wie selbstbewußt sich vor allem die jüngere Richtergeneration der neuen Lage stellt, zeigten der IV. Richterkongreß in Niamey 98 und die Gründung einer Richtergewerkschaft.

93 Dabei fehlt in der Verfassung 1992 die in Art. 67 GG erfolgte Limitienung auf ein konstruktives Mißtrauensvotum.

94 In der politischen Diskussion gab es weitergehend einige Stimmen für eine Bundesstaatslösung im Niger (vergleichbar mit dem südlichen Nachbarland Nigeria). Angesichts des sich verstärkenden Tuaregaufstands im Norden des Landes verloren sie im Sommer 1992 deutlich an Gewicht.

95 Die Nachwirkungen Montesquieuscher Gedanken (De l'esprit des lois) war hier deutlich spürbar, Näher: Laouel, Autres pouvoirs, conseils et organes constitutionnels, in: Sahel Dimanche v. 3.7.1992, S. 4.

96 Formeller Garant ist traditionell, aber jetzt systemfremd der Präsident der Republik (Art. 101 Abs. 2).

97 Conseil Supérieur de la Magistrature.

98 Vgl. den Tagungsbericht (Niamey 1992), passim. 
Die Gerichtsorganisation sieht über den Zivil-, Straf-, Arbeits- und Verwaltungsgerichten einen Obersten Gerichtshof 99 vor (Art. 104), der vier Senate für Verfassungs-, Zivil-/Straf-, Verwaltungs- und Rechnungsprüfungssachen umfaßt. Es wäre im Interesse des Verfassungs-/Rechtsstaats sachgerechter gewesen, ein eigenständiges Verfassungsgericht einzurichten.100 Aus Kostengründen und wegen vermuteter Nichtauslastung hatte aber bereits der Verfassungskonvent von Namaro darauf verzichtet.

Ubernommen in die Verfassung 1992 wurde dagegen die französische Fehlkonstruktion eines besonderen Hohen Gerichtshofs 101 aus Abgeordneten der Nationalversammlung. Er soll über Hochverrat102 des Präsidenten der Republik103 und über Regierungsmitglieder urteilen, die Vergehen oder Verbrechen im Amt begangen haben (Art. 106). Anklage wird in diesen besonderen Strafverfahren auf Beschluß der Nationalversammlung erhoben. Der Beschluß bedarf bei der Präsidentenanklage der Zweidrittel-, bei Regierungsmitgliedern der einfachen Mehrheit der gesetzlichen Mitglieder der Nationalversammlung. Bei der Tätigkeit des Hohen Gerichtshofs handelt es sich um eine Durchbrechung des Grundsatzes der Gewaltentrennung, der zur Gewaltenhemmung nicht geboten ist. Wie im Frankreich der V. Republik104 erwies sich auch im Niger der Übergangsphase105 im Jahre 1992 die Einrichtung eines Hohen Gerichtshofs als uneffektiv und schwerfällig.

\section{Räte als besondere Verfassungsorgane}

\section{a) Wirtschafts-, Sozial-und Kulturrat}

Die Verfassung 1992 erweiterte den im Niger seit der Staatsgründung, französischer Verfassungstradition folgend, geschaffenen Wirtschafts- und Sozialrat 106 zum Wirtschafts-, Sozial- und Kulturrat. Als Repräsentationsorgan berät das Gremium die anderen Staatsorgane im Hinblick auf die Interessen/Argumente der von ihm vertretenen Bevölkerungs-

99 Cour Suprême.

100 Das geschah z.B. in Benin, Burundi, Mali und Togo.

101 Haute Cour de Justice.

102 Dessen Definition findet sich in Ar. 106 Abs. 2: "Hochverrat liegt vor, wenn der Präsident der Republik seinen Amtseid bricht oder als Unheber, Mitverursacher oder Beteiligter an schweren Menschenrechtsverletzungen oder an unlauterer Abtretung eines Teils des Staatsgebiets (mit-) wirkt."

$103 \mathrm{Vgl}$. Ar. $61 \mathrm{GG}$.

104 Fall der kontaminierten Blutkonserven.

105 Anklage von zwei angeblich von Präsident Kountché zum Mord angestifteten Offizieren.

106 Im Niger kannte bereits die Verfassung von 1960 den Wirtschafts- und Sozialrat. Auch auf EGEbene hat man mit dem (beratenden) Wirtschafts- und Sozialausschuß (Ar. 193-198 EWGV) bisher recht positive Erfahrungen gemacht. Dagegen stehen negative Erfahrungen in Deutschland mit dem Reichswirschaftsrat in der Weimarer Zeit (Ar. 165 RV 1919 WRV). 
kreise. Der Rat unterstützt z.B. den Präsidenten der Republik, die Regierung und die Nationalversammlung durch Stellungnahmen zu Gesetzentwürfen und anderen Vorlagen zu wirtschaftlichen, sozialen und kulturellen Problemen. Er kann auch von sich aus Studien und Untersuchungen in den genannten Bereichen erstellen (lassen) und sie der Regierung zuleiten. Zusammensetzung, Organisation und Verfahren des Rats regelt ein noch zu erlassendes einfaches Gesetz (Art. 111). Nach dem Kommissionsbericht107 soll der Rat auch für eine gewisse regionale Repräsentation Sorge tragen.

\section{b) Medienrat}

Bereits in der Ubergangsphase arbeitete ein Medienrat108 und erleichterte den neu gegründeten (Wochen-) Zeitungen und dem öffentlich-rechtlichen, jetzt demokratisch orientierten Rundfunk (Hörfunk und Femsehen) den Start bzw. die Arbeit in dem gewandelten soziokulturellen Rahrnen und dem geänderten Medienverständnis. Aufgrund der kurzen, aber überwiegend positiven Erfahrungen schrieb die KGT den Medienrat in der Verfassung 1992 fest. Seine eigentliche Bewähnngsprobe als Sicherungsorgan für die Freiheit und Unabhängigkeit der Medien wird der neue Rat aber noch zu bestehen haben, wenn die gewählten Staatsorgane 1993 zu arbeiten beginnen.

\section{Gebietskörperschaften}

Den Gebietskörperschaften werden - wie in fruheren nigrischen Verfassungen - nur zwei Artikel des X. Abschnitts (Art. $115 \mathrm{f}$.) gewidmet. Das zeigt einmal mehr, wie schwer es dem Niger fällt, sich aus den französischen Rechtstraditionen zu lösen. Dabei wird im Land seit vielen Jahren über Dezentralisienung diskutiert. Immerhin wird in der Verfassung 1992 erstmals festgeschrieben, daß die Verwaltung des Territoriums auf den Grundsätzen von Dezentralisierung und Dekonzentration beruht (Art. 115 Abs. 1). Alle übrigen Regelungen, etwa die Begründung einer kommunalen Selbstverwaltung, werden dem Gesetzgeber zur Regelung durch einfaches Gesetz überlassen. Gerade angesichts regionaler Spannungen im Land ist das so weit gehende Schweigen des Verfassungsgebers zur - allseits gewünschten Stärkung der Kommunen schwer verständlich.

107 s.o. FN 35, hier: S. 7.

108 Conseil Supérieur de la Communication (CSC); der CSC organisiente z.B. im Herbst 1992 die Versammlung der Medienmitarbeiter (états généraux de la communication); näher: Laouel, Autres pouvoirs, conseils et organes constitutionnels, in: Sahel Dimanche v. 10.7.1992, S. 4. 


\section{Internationale Beziehungen}

Auch in der neuen Verfassung (Art. 117-121) erweist sich der Niger als international kooperatives Land und zuverlässiger Vertragspartner. Die afrikanische Einheit steht im Vordergrund der genannten Regelungen. Der Niger darf danach mit afrikanischen Staaten Assoziations- und Gemeinschaftsvertuäge abschließen und dafür partiell auf seine Souveränität verzichten. Art. 121 Abs. 1 geht sogar noch weiter und läßt zugunsten der Realisierung der afrikanischen Einheit einen totalen Souveränitätsverzicht zu. Ob eine solche Aufgabe der eigenen Staatlichkeit durch den Verfassungstext vorgesehen werden darf oder ob sie nicht vom Volk mit qualifizierter Mehrheit (oder gar einstimmig) beschlossen werden müßte, wurde in der KGT nur kurz diskutiert.

\section{Verfassungsänderungen, Übergangs- und Schlußbestimmungen}

Nach Art. 122 dürfen der Präsident der Republik auf Vorschlag des Premierministers und Mitglieder der Nationalversammlung die Gesetzesinitiative zur Verfassungsänderung ergreifen. Der Entwurf bedarf, um Gesetz zu werden, einer Dreiviertelmehrheit der gesetzlichen Mitglieder der Nationalversammlung und ist einem Referendum zu unterwerfen, wenn er nicht von Vierfünfteln der gesetzlichen Mitglieder der Nationalversammlung angenommen wurde.

Die Verfassung 1992 trat mit der Verkündung durch den Präsidenten der Republik während der acht Tage nach der Bekanntgabe des Ergebnisses der Volksabstimmung vom 26. Dezember 1992 in Kraft. Der Akt XXI der Nationalkonferenz109 blieb für die Organisation der Staatsgewalt bis zum Amtsantritt der gewählten Organe (Organwalter) in Kraft (Art. 127 Abs. 1). In Absatz 2 von Art. 127 wird das Verbot bezüglich der Spitzen der Transitionsorgane (Präsident der Republik, Präsident des HRR und Premierminister) ausgesprochen, 1993 für das Präsidentenamt zu kandidieren. 110

\section{Zusammenfassung und Ausblick}

Die neue Verfassung der Republik Niger entstand unter Zeitdruck. Ihre Entstehung war eingebettet in die nur 16monatige Ubergangszeit von Ausnahmeregime und Diktatur zu demokratisch gewählten/legitimierten Staatsorganen. Trotz guten Willens innerhalb der

109 Dazu näher: Lqaouel / Aichatou, Les lacunes juridiques de l' Acte Fondamental No. XXI portant organisation des pouvoirs publics, in: Sahel Dimanche v. 17.7.1992, S. 4; Issoufou, La Transition malade de son droit, in: Anfani v. 5.8.1992, S. 4 f.

110 s.o. FN 90. 
KGT und des HRR und des dort versammelten Sachverstands enthält der Text einzelne Ungereimtheiten, unzweckmäßige Lösungen und Lücken, die oben dargestellt wurden. Die sehr starke Stellung des Präsidenten der Republik, die entsprechend schwächere des Premierministers/der Regierung und des Parlaments, Ungenauigkeiten bei der Kompetenzabgrenzung, zu weit und unpräzise gefaßte Bildungs-, Umwelt- und Widerstandsrechte, das Verhältniswahlrecht ohne Sperrklausel (z.B. von $5 \%$ ), das Fehlen einer Regionalkammer des Parlaments und eines eigenständigen Verfassungsgerichts sowie die nicht hinreichende Ausgestaltung der Stellung der Kommunen gehören dazu.

Dem stehen die verstärkte Demokratie- und Gewaltenteilungskomponente (letztere im Sinne einer Gewaltenhemmung), die friedliche Ablösung des Einparteiensystems, der Ausbau des Rechtsstaates generell und der Grundrechte, der Versuch der Förderung unabhängiger Medien, der Bekämpfung der Korruption, des Abbaus ethnischer und religiöser Spannungen gegenüber. Insgesamt stellt die Verfassung 1992 einen bedeutenden Fortschritt für die Nigrer und den nigrischen Staat dar. Sollte es gelingen, den Verfassungstext in die Praxis umzusetzen, trägt er sogar den Keim eines Verfassungspatriotismus in sich. 


\section{ABSTRACTS}

\section{A False Start: Law and Development in the Context of a Colonial Legacy}

\section{By Bas de Gaay Fortman and Paschal Mihyo}

The article reflects upon the development of law in former colonies in Africa both during the period of colonialism and after independence had been reached. Law is seen and analyzed as part of a social process, and in this perspective the impact of political and economic colonial strategies upon the structures and institutions of pre-existing African laws is described, focussing on the legislative process, the function of norms in African communities, the settlement of disputes and the role of sanctions. The article then turns to criticize the way in which post-independence societies made use of relicts of the colonial legal system, regarding law as a major instrument of social change and neglecting elements of traditional law. It defines the legal legacy of colonisation as a major constraint to peoplecentered development in the post-independence period, and thus as a "false start" rooted in structures long before independence.

Consequently, the authors call for an unmaking of existing state policies and laws in specific legislative areas.

\section{Niger: The New Constitution of the Third Republic}

\section{By Volker Lohse}

In spite of social tensions due to the country's poor economic and financial situation and due to a massacre among the Tuareg in the north, the West African Republic of Niger has made a courageous step forward towards democratization by substituting the state of emergency of the Second Republic with the new Third Republic. The new Constitution (Constitution de la III République) which has been accepted by the referendum of December 26,1992 is the new foundation for more participation of the citizens, the multi-party system and the rule of law.

The new constitution is partly modelled after the French constitution of the Fif th Republic. It follows, however, an independent path, e.g., with the distribution of executive powers among the president, the prime minister and the cabinet and by strengthening of the judiciary and including a bill of rights. 
Since its coming into force, the constitution proved effective in the democratic election of February 1992 and the subsequent inauguration of the members of the main institution of the state.

\section{Societal Scripts and Constitutional Law as Competing Basis for Political Order of Ethiopia}

\section{By Heinrich Scholler}

The Transitional Constitution of Ethiopia (transitional period charter of Ethiopia of 22nd July 1991) states in Art. 2 that the different National Societies, the central and the regional powers have to decide to what extent they will adopt modern constitutional law or recur to traditional organisation of their societies.

The Societal Script is the expression of internal not-written political order, which has to be taken into consideration. Scholars have put emphasis on the fact that the Societal Script of different groups like the Tigray-amhara and the Oromo form a thesis and anti-thesis as vertical and horizontal structure in Society, which have to be reconciled. These competing structures of the different Societal Scripts could be organised in a federal system for allowing to have diversity on the regional and cultural level together with national or central unity. Modem and traditional law (Societal Scripts) must not remain an unsolved conflict.

In the Context of the Current Developments in African Constitutional Law: The Adventure of the National Conference in Togo

By EA.B. van Rouveroy van Nieuwaal

During the last hundred years, Africa has seen profound political and constitutional changes the extent of which is closely linked to the respective colonial power. The African state is a juridical entity in international law, but was it also, at the time of independence, an empirical entity?

In almost all cases the newly independent states in Africa kept a number of the European administrative officials who formerly represented the colonial state. Independence, therefore, created a gap between the international legitimacy and the internal legitimacy of many African states. 